\title{
Situación actual de la planeación curricular en los centros de formación superior de El Salvador
}

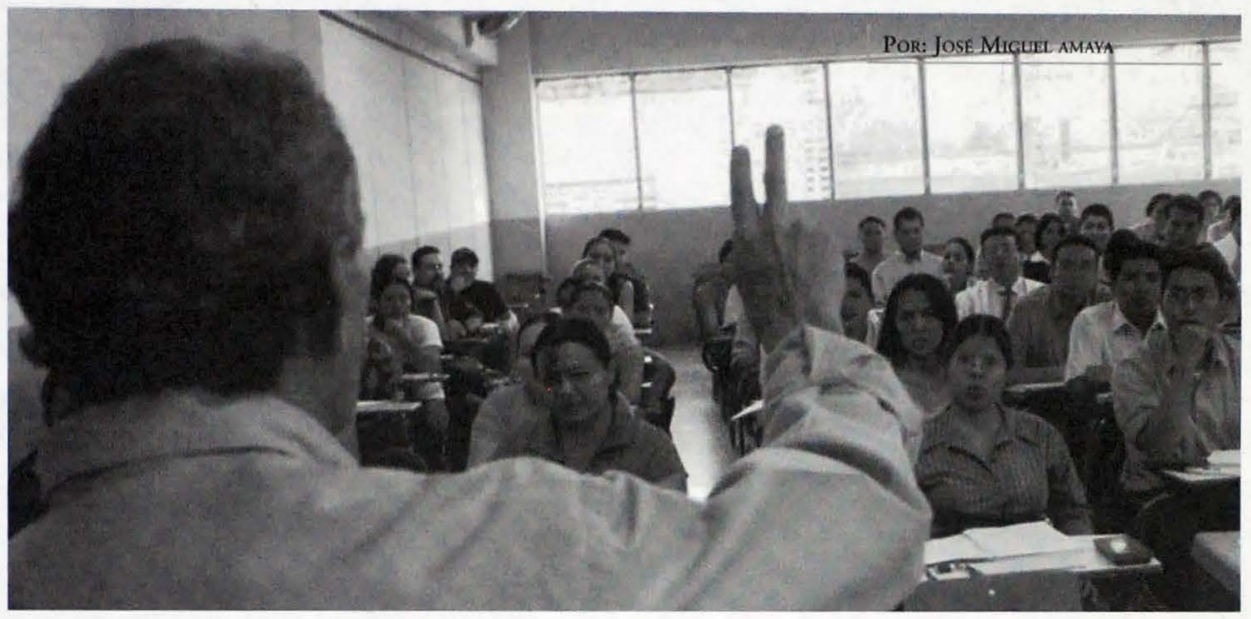

A continuación se presenta el análisis cuantitativo de los datos encontrados en el estudio realizado, la presentación se realiza por rubros analizados determinando numéricamente los hallazgos.

\section{Dimensiones de la evaluación curricular}

En este primer rubro de análisis puede notarse, que los catorce factores consultados a los maestros y maestras de las universidades reflejan según los datos generales situaciones muy deficitarias, que podrían estar incidiendo de manera negativa en la búsqueda de la calidad y la excelencia académica a la que aspira toda institución de educación superior en El Salvador. Si observamos con atención cada una de las áreas consultadas identifican con certeza y con mucha cobertura los elementos que deben considerarse en el quehacer curricular de las universidades y cada uno debe verse reflejado en el currículo elaborado para cada una de las carreras que se ofertan al conglomerado estudiantil.

Los datos encontrados son el claro reflejo de la problemática que enfrentan las universidades en lo correspondiente al planeamiento curricular del área a la cual no han prestado la debida atención, en tanto la preocupación de esta ha estado centrada en la docencia y no en la responsabilidad que tienen como instituciones educativas de garantizar que los servicios que se prestan estén debidamente consultados y orientados a la satisfacción de las necesidades y problemas que enfrenta la sociedad en su conjunto. Por ello, los currículos que las instituciones de educación superior ofrecen deben estar debidamente elaborados y consensuados con los distintos actores de la realidad nacional, para que el estudiante egresado pueda integrarse satisfactoriamente al quehacer transformador de la sociedad misma.

En términos generales podemos sostener que solamente el 28 por ciento de los consultados con respecto a estos catorce factores consideran que existe en las instituciones de educación superior un proceso por medio del cual la curricula esta siendo desarrollada según las exigencias técnicas debidas; pero, el 72 por ciento sostiene que esta actividad en las instituciones es signifi-cativamente deficitaria y que requiere que se le asigne un nivel de interés particular.

\section{Elementos doctrinarios de la evaluación curricular}

Los elementos doctrinarios de la planeación curricular en las instituciones de educación superior de El Salvador, según la opinión obtenida por medio de los consultados, no es una fortaleza que prima en la calidad de la construcción del currículo. En el diagnóstico de la realidad, como elemento primordial para la elaboración del currículo, se revisan las demandas del proceso productivo y el desarrollo del país, para que por medio del currículo, se pueda dar respuestas integrales a los estudiantes para 
que se integren a una realidad que conocen desde la universidad, responde a las motivaciones e intereses y expectativas permanentes de los estudiantes, en tanto esta basada en las necesidades, intereses y problemas que el estudiante considera pertinentes que deben ser analizados desde la palestra universitaria.

El debate periódico, con la participación de todos los estamentos, se promueve en torno al currículo un análisis constructivo de los planes y programas de la carrera para estar realimentando de manera permanente y sostenida el currículo propuesto, preveer experiencias que demuestren a los educandos el nuevo tipo de sociedad que se desea. Los elementos planteados en el párrafo anterior orientan la situación actual del planeamiento curricular con respecto a los elementos doctrinarios y los datos son muy reveladores en tanto solamente el $30 \%$ de los consultados consideran que estos son realizados por los encargados del currículo en las universidades y el $70 \%$ considera que estos elementos son muy deficitarios en el quehacer curricular de las instituciones de educación superior.

\section{Coherencia del curriculo institucional}

Es necesario en la estructuración del currículo definir con mucha claridad la coherencia que este posee y por ello ha sido necesario revisar este aspecto en la investigación. La consulta refleja que en la mayoría de universidades del país el currículo responde más a situaciones institucionales y el cumplimiento de requisitos demandados por entes contralores como el Ministerio de Educación y no a dar respuestas coherentes a las grandes necesidades que los estudiantes, empresas, y sociedad en general requieren; esto deja en claro que se tiene un currículo educativo en las universidades que está alejado del quehacer real de las instancias que al final requerirán los servicios de los egresados.
Los hallazgos del estudio determinan que solamente el 25 por ciento del currículo, según apreciación de los consultados tiene coherencia con los objerivos, los contenidos, las formas de enseñanza y el sistema de evaluación, ausencias de contenidos esenciales, falta de vinculación entre disciplinas y asignaturas, la planeación curricular está desvinculada a la enseñanza que requiere el estudiante. El 75 por ciento considera que todos estos elementos están formulados sin coherencia en el currículo de las universidades del país o por lo menos en la universidad donde trabajan.

\section{Métodos de globalización del curriculo}

La educación moderna tiene como exigencia la necesidad de que su currículo este integrado con todos los actores de la sociedad. Para ello, es necesario considerar al currículo como un evento globalizador que permite la participación de todos los interesados en que la educación se cualifique en todos sus aspectos. Los contenidos, planes y programas no deberían admitir que se genere ningún tipo de fragmentación. Según los datos encontrados en el estudio, una cantidad poco significativa es decir el 26 por ciento de los consultados sostiene que si existe un interés por la globalización del currículo, pero un 74 por ciento determina que no existe, ni se vislumbra en los currículos de las universidades elementos que permitan sostener que hay un interés real de las instancias encargadas de iniciar un proceso de consulta que responda a los intereses de los estudiantes.

\section{Evaluación curricular y contexto}

El proceso de la evaluación curricular debería en las universidades de El Salvador estar orientado por los cuatro aspectos que se plantean en el contexto anterior, estos elementos deben ser considerados como esenciales en el currículo especialmente cuando se desee evaluar el contexto del currículo. La evaluación

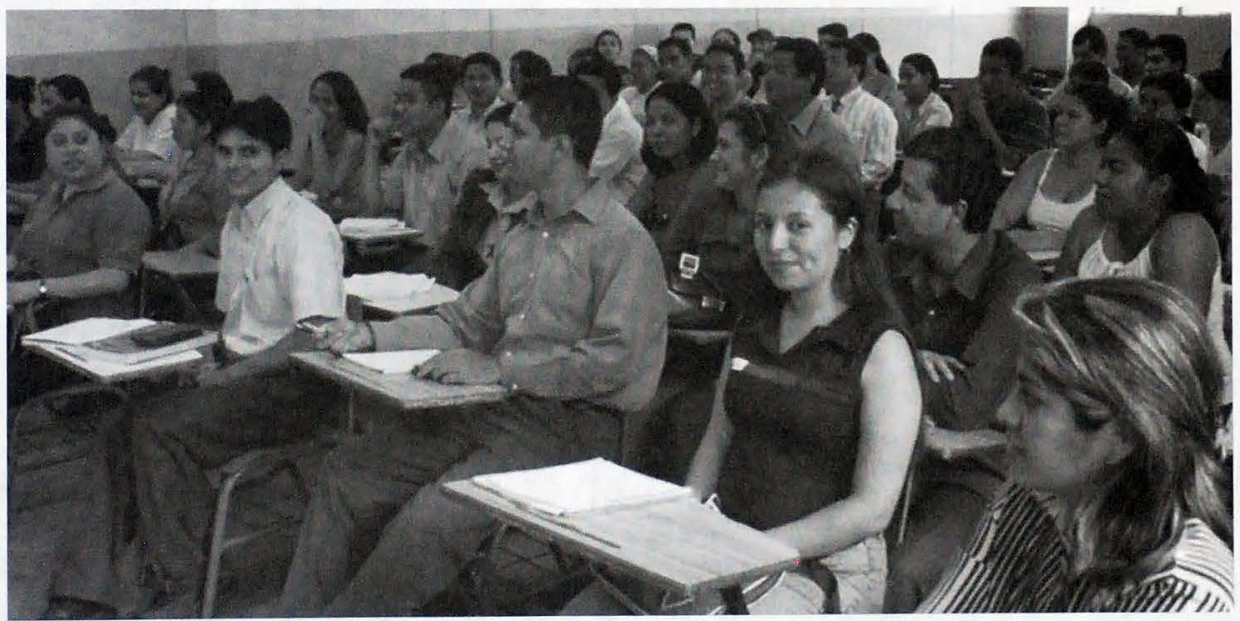


del contexto y la evaluación de insumo o entrada deben convertirse en prioridades para saber cual es el recorrido que debe realizarse en el proceso.

La evaluación de proceso y producto debe estar encaminada al seguimiento y control que se ejerce sobre la planeación misma. Estos cuacro elementos vitales del quehacer curricular en la universidades de cualquier parte del mundo, reflejan en el caso particular de El Salvador una situación sumamente incipiente ya que los datos obligan a determinar que la evaluación curricular de contexto no esta siendo realizada. Solamente en el 22 por ciento de los consultados observan que si existe.

\section{Evaluación de la eficiencia curricular Congruencia}

Los datos reflejan que los procesos curriculares cuando se refieren a la evaluación de la eficiencia, por lo general, se le presentan a un grupo reducido de profesionales, para que estos emitan sus juicios de expertos y con ello se resuelven los problemas. Esto demuestra que no existe en este aspecto un interés decidido por parte de las instituciones de apostarle a una

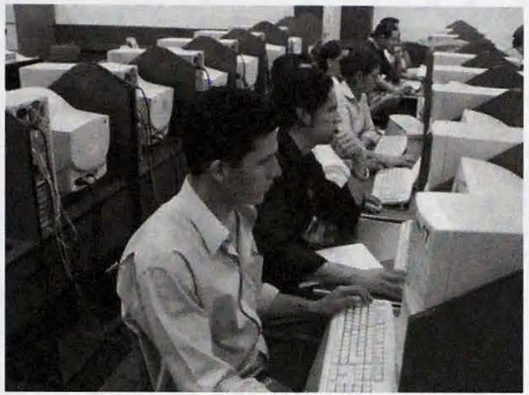

consultados, el 73 por ciento, determinan que no se está prestando debidamente la atención a este aspecto curricular.

\section{Evaluación de la eficiencia curricular Continuidad}

La continuidad curricular es imperativamente necesaria en los procesos, ya que por normativa jurídica por decisión institucional, y en última instancia, por necesidad y control institucional debería permitirse que los procesos curriculares operen de manera debida, así debe reconocerse que un currículo no debe estar orientado al asistencialismo, sino proyectarse a las áreas de investigación y eficientemente a la gestión profesional. También se permite que exista por medio del currículo una integración del docente con la realidad objetiva, usando los encargados del currículo estos elementos para que sea la base de la continuidad curricular. Los datos encontrados concluyen que esta área también presenta deficiencias reales en tanto los consultados sostienen que la continuidad del currículo esta orientada en gran medida al asistencialismo el 75 por ciento y existe una orientación a las áreas exigidas como son la investigación y la gestión profesional. transformación eficaz de la manera

de elaborar el currículo. Cuando se cuanrifican los datos del estudio, solamente un 29 por ciento, considera que cuando se evalúa el currículo en su congruencia se toman en cuenta los aspectos evaluados en este estudio.

\section{Evaluación de la eficiencia curricular Viabilidad}

Estos aspectos denotan en el análisis también una deuda con la realidad ya que los participantes en el estudio consideran que todavía no se esta garantizando la viabilidad curricular en los centros de formación superior en El Salvador. Solamente un 29 por ciento sostiene que existen esfuerzos para garantizar la viabilidad curricular en las universidades del país.

\section{Evaluación de la eficiencia curricular Vigencia}

La evaluación de la eficiencia curricular específicamente en su vigencia es necesaria para identificar de manera particular aquellos elementos que por lo general se plantean de manera sumamente idealizada. Por lo general, distanciados del diseño del currículo que necesitan los estudiantes para responder a las exigencias de los entornos profesionales; $y$ por otra parte, las asignaturas servidas en cada una de las carreras que atiende la institución deben reflejar la eficiencia y la eficacia del plan propuesto y con ello garantizar la formación integral que requiere el estudiante. Los resultados que se detallan en el cuadro de vaciado de datos reflejan una clara situación de desfavorecimiento en el aspecto planteado de la vigencia del currículo, ya que un número significativo de
A modo de recomendaciones para las instituciones de educación superior en El Salvador se presentan las siguientes:

1. Las instituciones de educación superior deben realizar un proceso sistémico para elaborar sus planes y programas de estudio y estos deben garantizar la participación activa de todos los sectores de la sociedad nacional, este proceso deberá ser parte inherente del quehacer de la institución y debe permitir la cualificación de los procesos educativos en todas sus dimensiones. Por tanto, se recomienda crear una instancia colegiada con una política curricular definida para que cada uno de los procesos de revisión, actualización y seguimiento de los planes y programas de estudio en cada una de las carreras que la institución atiende estén determinados por un proceso democrático y participativo que garantice la calidad, pertinencia y novedad del currículo propuesto.

2. Es imperativo necesario, que las universidades del país elaboren una política curricular que responda a los más altos intereses de la misión u visión de las institución y con ello garantizar que en los planes y programas de estudio que se oferten estén incorporados aquellos principios Teleológicos, Axiológicos y Deontológicos que permitan integrar en cada una de sus fases. Para diseñar el currículo: la investigación, que le abre los horizontes posibles, la orientación, que le precisa el horizonte a elegir y el diseño propiamente dicho, que articula anticipadamente las acciones que harán posible el logro del horizonte elegido. Para 
producir los aprendizajes constitutivos del currículo: la implementación, que permite contar con las condiciones necesarias para tal aprendizaje y la ejecución, que constituye el proceso mismo de logro. Para evaluar, el monitoreo y evaluación, que informa sobre el proceso y los resultados.

3. El cambio curricular en el desarrollo institucional es una necesidad vital en los centros de formación superior y este puede sustentarse con la afirmación de que «el cambio curricular es una variedad de cambio educativo, que a su vez, es una forma de cambio socials Cultura educativa y cambio curricular. Hoy, sin embargo, entendemos que en último extremo el cruce de factores sociales, políticos y práctica educativa se juega en esos espacios institucionales de mediación que son los centros de formación superior, donde se configuran los procesos de enseñanza aprendizaje de los alumnos y profesores. Por eso vamos a defender que no cabe esperar una mejora educativa desconectada de las condiciones internas del centro, por lo que cualquier reforma curricular impuesta externamente, si quiere ser exitosa, tendrá que ser reconstruida por la institución de educación superior de acuerdo con sus prioridades; al tiempo de ir creando condiciones internas

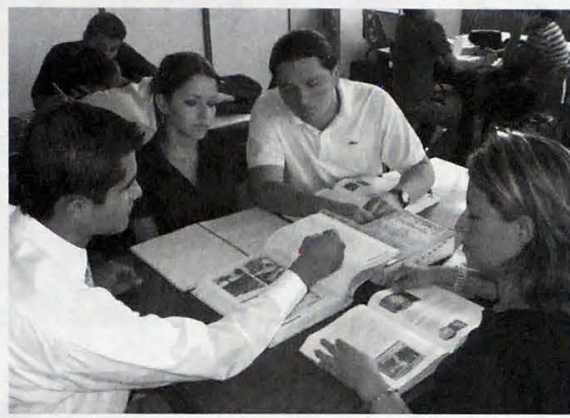

para propiciar el trabajo en equipo y la coordinación vertical y horizontal. Promueva la participación de las comisiones curriculares en los procesos de diseño, ejecución, evaluación y rediseńo de los planes de estudio que se desarrollan en forma compartida entre unidades académicas. Promueva la articulación entre los programas, proyectos y actividades de investigación, extensión, producción y docencia, mediante los cuales se involucre a estudiantes, docentes y comunidad en general. Asegure la creación de condiciones óptimas para ofrecer diversas modalidades educativas, mediante la incorporación de nuevas tecnologías en la docencia universitaria, tales como educación a distancia y educación virtual. Garantice procesos formativos para que la población estudiantil en toda su diversidad, tenga acceso a la formación académica universitaria, considerando el perfil de ingreso de cada carrera.

5. Se recomienda a las instituciones de educación superior que elaboren con claridad y conocimiento los lineamientos curriculares que permitan orientar el currículo institucional por senderos que permitan. Los lineamientos constituyen una toma de decisión para cumplir con la política respectiva, y resolver las necesidades detectadas en materia curricular. Estos lineamientos se y externas para provocar un desarrollo organizativo o institucional.

4. Garantice la coordinación curricular permanente entre unidades académicas que diseñen carreras con objetos de estudio o áreas de conocimiento similares. Presente estructuras de organización curricular flexibles, acordes con las características de la institución y de la población meta a la que se dirige. Mantenga una vigencia definida con base en criterios de matrícula, graduación, pertinencia social y laboral. Integre el aspecto interdisciplinario, pluridisciplinario y transdisciplinario de los objetos de estudio. Incorpore un eje de investigación que impregne cada uno de los cursos del plan de estudios de las carreras que se ofrezcan, con el fin de formar al estudiante bajo el principio que el aprendizaje no termina al concluir el curso o carrera, sino que es su responsabilidad como profesional seguir aprendiendo mediante la investigación permanente de su objeto de estudio. Incorpore enfoques y metodologías que promuevan la integración de la teoría con la práctica, mediante conocimientos y herramientas tecnológicas actualizadas, según las particularidades de cada carrera.

Integre, además, acciones de gestión y administración curricular en términos de organización, ejecución, evaluación y control, esbozan en 5 áreas temáticas: a. diseńo y planificación curriculares, b. organización del contenido curricular, c. evaluación curricular, d. administración curricular y e. apoyos para el desarrollo curricular institucional. Esta separación obedece únicamente a razones de organización, ya que ellas conforman una sola unidad.

6. Deben las universidades desarrollar lineamiento u orientación curricular para garantizar que la toma de decisiones este sustentada en las más altas aspiraciones de la institución de educación superior que realiza el desarrollo y la promoción curricular. Constituye una toma de decisión que define una acción específica para cumplir con la política respectiva y abordar la cuestión señalada como problema en el campo curricular. La flexibilidad curricular es una propuesta alternativa a la concepción lineal y rígida de la formación de profesionales. Bajo un enfoque holístico, pretende rescatar y poner en práctica la formación integral y autónoma del estudiante. Entre otros aspectos posibilita ampliar y diversificar las opciones de formación profesional conjugando intereses y necesidades personales, profesionales e institucionales; la incorporación sistemática de nuevos conocimientos que se derivan del avance disciplinar y de las características del entorno; la formación inter, multi y transdiciplinaria, así como opciones diversas en los grados y títulos que se otorgan. 